Manuelle Medizin 2012 · 50:101

DOI 10.1007/s00337-012-0912-9

Online publiziert: 17. April 2012

(c) Springer-Verlag 2012

L. Beyer

Geschäftsstelle DGMM, Ärztehaus Mitte, Jena

\title{
Wissenschaftliche Erkenntnisse unterliegen einem Erneuerungsprozess
}

tikel. In einem ganzheitlichen funktionellen Gefüge wirken sich Änderungen eines Parameters auf andere Parameter aus. Um die Zusammenhänge zu verstehen, müssen wir einzelne Aspekte tiefer analysieren. In diesem Heft widmet sich der Beitrag von Böhni et al. der biomechanischen Seite der Dysfunktion, bezogen auf periphere und Wirbelsäulengelenke, immer in Relation zu muskulären Befunden und Schmerzempfindungen. So führt die Änderung von biomechanischen Parametern am Wirbelsäulensegment zu veränderten Bewegungsmustern und einer vermehrten mechanisch-strukturellen segmentalen Beweglichkeit. Diese umfassende Auseinandersetzung mit biomechanischen Aspekten trägt zur Interpretation der Wirkungsweise und zum differenzierten Einsatz manualmedizischer Behandlungstechniken bei. Ein weiterer Beitrag dieses Hefts beschäftigt sich mit dem Zusammenhang zwischen Rückenschmerz und der Stabilität der lumbalen Wirbelsäule in der Schwangerschaft.

\section{》) Immer wieder neu durchdenken, immer wieder neu verstehen}

Wissenschaftliche Fakten unterliegen einem Erneuerungsprozess, neue Fakten lassen ältere Hypothesen überholt erscheinen oder lösen diese ab - so erneuert sich auch Evidenz. Die in den Beiträgen zur Wirksamkeit der „Manualthe- rapie bei chronischem Spannungskopfschmerz“, „Okklusionsstörungen und die Auswirkungen auf den funktionellen Bewegungsraum der Lendenwirbelsäule "sowie „Muskuloskeletale Befunderhebung unter manualmedizinischen Aspekten im Schulter-Nacken-Bereich bei 12-jährigen Schülern" (MM aktuell) aufgezeigten Ergebnisse sind unter diesem Aspekt $\mathrm{zu}$ sehen. In Abwandlung eines geflügelten Wortes in der Quantenphysik könnte man fast sagen: „Wer behauptet die manuelle Medizin zu verstehen, hat sie nicht verstanden.“ Mit anderen Worten: „immer wieder neu durchdenken, immer wieder neu verstehen" - ein schönes Motto für den Frühling.

Mit freundlichen Grüßen

Ihr

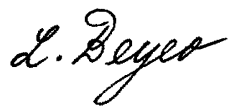

L. Beyer

\section{Korrespondenzadresse}

Prof. Dr. L. Beyer

Geschäftsstelle DGMM, Ärztehaus Mitte, Westbahnhofstr. 2, 07745 Jena lobeyer@t-online.de 